\title{
Supercritical carbon dioxide-developed silk fibroin nanoplatform for smart colon cancer therapy
}

\author{
This article was published in the following Dove Press journal: \\ International Journal of Nanomedicine \\ 20 October 2017 \\ Number of times this article has been viewed
}

\author{
Maobin $\mathrm{Xie}^{1,2, *}$ \\ Dejun Fan ${ }^{3, *}$ \\ Yi Li ${ }^{4}$ \\ Xiaowen $\mathrm{He}^{3}$ \\ Xiaoming Chen ${ }^{1,2}$ \\ Yufeng Chen ${ }^{3}$ \\ Jixiang Zhu $u^{1,2}$ \\ Guibin $X u^{5}$ \\ Xiaojian $\mathrm{Wu}^{3}$ \\ Ping Lan ${ }^{3}$
}

'School of Basic Medical Sciences, Guangzhou Medical University, Guangzhou, China; ${ }^{2}$ Key Laboratory of Oral Medicine, Guangzhou Institute of Oral Disease, Affiliated Stomatology Hospital of Guangzhou Medical University, Guangzhou, China; ${ }^{3}$ Department of Colorectal Surgery, Sixth Affiliated Hospital of Sun Yat-Sen University, Guangzhou, China; ${ }^{4}$ School of Materials, University of Manchester, Manchester, UK; ${ }^{5}$ Department of Urology, Fifth Affiliated Hospital of Guangzhou Medical University, Guangzhou, China

*These authors contributed equally to this work

Correspondence: Yi Li

School of Materials, University

of Manchester, Oxford Road,

Manchester MI 3 9PL, UK

Tel +44 I6I 3062676

Fax +44 $79464 I 8378$

Email henry.yili@manchester.ac.uk

Ping Lan

Department of Colorectal Surgery, Sixth Affiliated Hospital of Sun Yat-Sen

University, 26 Yuancun Erheng Road,

Guangzhou, Guangdong 510655, China

Tel +86203825 580I

Fax +86 203825422 I

Email lanping@mail.sysu.edu.cn
Purpose: To deliver insoluble natural compounds into colon cancer cells in a controlled fashion.

Materials and methods: Curcumin (CM)-silk fibroin (SF) nanoparticles (NPs) were prepared by solution-enhanced dispersion by supercritical $\mathrm{CO}_{2}$ (SEDS) (20 MPa pressure, 1:2 CM:SF ratio, $1 \%$ concentration), and their physicochemical properties, intracellular uptake efficiency, in vitro anticancer effect, toxicity, and mechanisms were evaluated and analyzed.

Results: CM-SF NPs $(<100 \mathrm{~nm})$ with controllable particle size were prepared by SEDS. CM-SF NPs had a time-dependent intracellular uptake ability, which led to an improved inhibition effect on colon cancer cells. Interestingly, the anticancer effect of CM-SF NPs was improved, while the side effect on normal human colon mucosal epithelial cells was reduced by a concentration of $\sim 10 \mu \mathrm{g} / \mathrm{mL}$. The anticancer mechanism involves cell-cycle arrest in the $G_{0} / G_{1}$ and $G_{2} / M$ phases in association with inducing apoptotic cells.

Conclusion: The natural compound-loaded SF nanoplatform prepared by SEDS indicates promising colon cancer-therapy potential.

Keywords: supercritical, silk fibroin, nanoparticle, natural compounds, colon cancer

\section{Introduction}

Curcumin $(\mathrm{CM})$ is a natural polyphenolic compound obtained from the root of Curcuma longa Linn (turmeric). ${ }^{1}$ It is reported that CM possesses multiple biomedical functions, such as antibacterial, antioxidant, anticancer, anti-inflammatory, anticarcinogenic, antispasmodic, and anticoagulant properties. ${ }^{2-4}$ Importantly, oral administration of CM $12 \mathrm{~g} /$ day exhibited little toxicity in clinical trials. ${ }^{5}$ However, clinical applications of insoluble $\mathrm{CM}$ face more challenging issues, such as low dissolution rate, low bioavailability, and controlled delivery.

Micronization is an effective strategy to overcome these drawbacks, such as with nanoprecipitation and spray-drying. ${ }^{6}$ However, these conventional micronization methods are always limited by organic solvent residuals and extreme processing conditions (such as high temperature). Owing to advantages such as mild process conditions $\left(\mathrm{T}_{\mathrm{c}}=304.1 \mathrm{~K}, \mathrm{P}_{\mathrm{c}}=7.38 \mathrm{MPa}\right.$ ), no organic solvent residues, and being environmentally benign, supercritical (sc) $\mathrm{CO}_{2}$ has been used in preparing nanoplatforms recently.,8 Among several sc- $\mathrm{CO}_{2}$ techniques, solution-enhanced dispersion by sc- $\mathrm{CO}_{2}$ (SEDS) emerges as a novel nanotechnology due to its broad range in selection of biomaterials and drugs. In SEDS, a special coaxial nozzle is used to produce very small droplets and improve the mixing effect between compound solution and $\mathrm{sc}-\mathrm{CO}_{2}$ flow, in order to promote mass transfer. ${ }^{9}$ Therefore, the surface morphology and particle size of the obtained particles can be controlled by parameters easily. ${ }^{10,11}$ Our group prepared CM 
NPs using SEDS technology, ${ }^{12}$ and results showed that CM nanoparticles (NPs) exhibited higher solubility and dissolution than original CM. Also, the CM NPs obtained showed effective biomedical functions. ${ }^{13}$

In order to control drug release, biomaterials are introduced to prepare polymeric nanoplatfoms. ${ }^{14-16}$ However, synthetic polymers may induce biocompatibility concerns. Natural silk is a type of fiber polymer which consists of glue-like silk sericin covering silk fibroin (SF). ${ }^{17} \mathrm{SF}$ is an excellent biomaterial, due to its good biocompatibility, ready availability, and controllable degradability. ${ }^{18-20} \mathrm{~A}$ recent study showed that tumors were noticeably reduced after being injected with $\mathrm{CM} / 5$-fluorouracil (Fu)-entrapped SF NPs. ${ }^{21}$ However, the mechanism to deliver incorporated drugs from SF nanoplatforms in a controlled fashion needs further investigation. Furthermore, traditional technologies to prepare SF NPs would induce protein degradation or drug volume loss.

In this study, CM-SF NPs were prepared by SEDS and the physicochemical properties of the CM-SF NPs prepared were tested. Intracellular uptake activity was visualized to study the interactions between CM-SF NPs and cancer cells. The in vitro viability of colon cancer cells and normal human colon mucosal epithelial cells when treated with CM-SF NPs was evaluated. Cell-cycle arrest and apoptosis assays were performed to investigate the mechanism of the anticancer effect of CM-SF NPs.

\section{Materials and methods Materials}

$\mathrm{CO}_{2}$ was from Hong Kong Specialty Gases (Hong Kong). Raw silk fibers of Bombyx mori were from the Jiangsu Wujing China Eastern Silk Market (Suzhou, China). Hexafluoroisopropanol (HFIP) was purchased from Huifengda Chemical (Jinan, China). Curcumin was from International Laboratory USA (San Francisco, CA, USA). Both human colorectal cancer cell line HCT116 and normal human colon mucosal epithelial cell line NCM460 were supplied from the cell bank of the Chinese Academy of Sciences (Shanghai, China).

\section{Preparation of curcumin-silk fibroin nanoparticles}

The procedure for preparing CM-SF NPs was based on our previous study, ${ }^{9}$ which is shown in Figure 1. Materials and procedure details are explained in the Supplementary material. Briefly, SF and CM were mixed and dissolved in hexafluoroisopropanol to the desired concentration (1\%). After dissolving completely, the blend solution was ready for the SEDS process (Figure S1).

\section{Physicochemical characterization}

To assess surface morphology, the prepared samples were observed via field-emission scanning electron microscopy (SEM, JSM-6490; JEOL, Tokyo, Japan). For particle size and size distribution, the SEM images were analyzed with Nano Measurer software and about 500 particles were measured., ${ }^{9,13}$ Molecular structure change was tested using Fourier-transform infrared spectroscopy (FTIR, 1720 spectrophotometer; PerkinElmer, Waltham, MA, USA). Preparation details are provided in the Supplementary material.

\section{Solubility test}

Solubility testing was conducted in accordance with our previous studies, with minor modifications. ${ }^{9}{ }^{913}$ Briefly, $10 \mathrm{~mL}$ PBS solution ( $\mathrm{pH}$ 7.4) was added to dry CM-SF NPs and original CM (10 mg) powders and the solution was kept under mild agitation (60 rpm). Insoluble residues were filtered after 1 hour, and then the concentration of the samples was detected at $410 \mathrm{~nm}$ using ultraviolet spectrometry for calculating solubility (Figure S2).

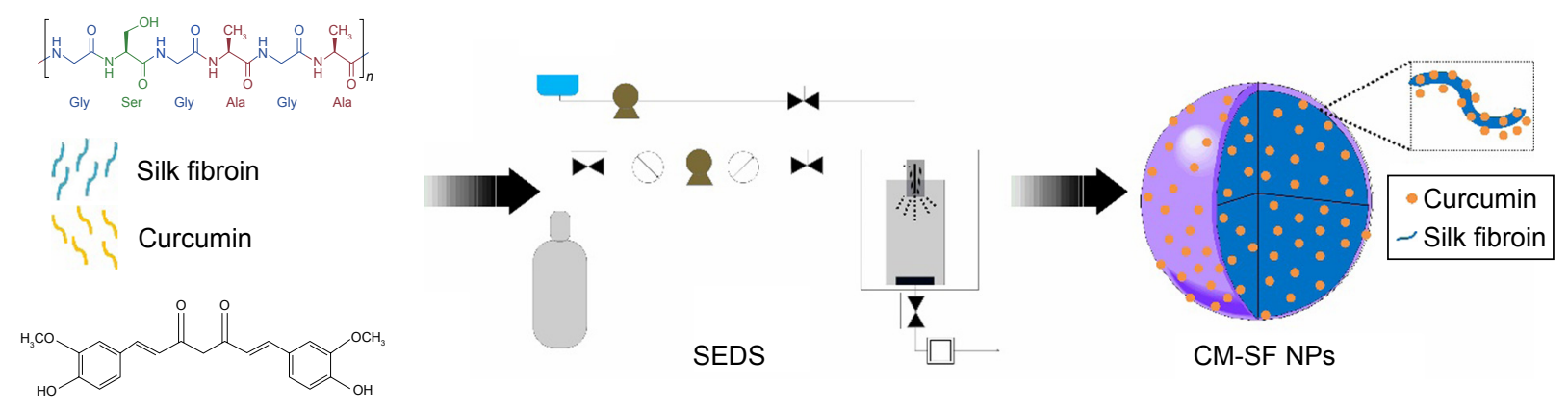

Figure I Preparation process of CM-SF NPs via SEDS.

Notes: SF powder and CM powder were mixed and dissolved in HFIP solution, ready for the SEDS process. After complete removal of HFIP solution, CM-SF NPs were collected. Yellow curves and circles, curcumin; blue curves, silk fibroin; blue oval, HFIP.

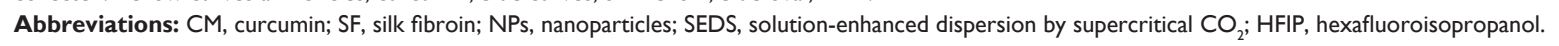




\section{Cellular uptake study}

All cell lines used in our research were purchased commercially from the cell bank of the Chinese Academy of Sciences (Shanghai, China). Procedures were based on our previous studies. ${ }^{13,22}$ First, $5 \times 10^{4}$ HCT116 cells were seeded onto coverslips in 24-well plates for cell attachment. Then, cells were treated with samples for 0.5 and 6 hours. After that, cells were washed with PBS and fixed with $4 \%$ formaldehyde for 1 minute. Secondly, formaldehyde was removed completely and $500 \mu \mathrm{L}$ methanol $\left(-20^{\circ} \mathrm{C}\right)$ was added, followed by 10 minutes' permeabilization with $0.2 \%$ Triton X-100 in PBS and washed with pure PBS. Thirdly, cells were sealed with $10 \%$ bovine serum albumin. Fourthly, cell nuclei were stained for 30 minutes with $5 \mu \mathrm{L}$ 4',6-diamidino-2-phenylindole. Lastly, stained cells were observed using confocal laser-scanning microscopy (TCS SP8; Leica Microsystems, Wetzlar, Germany).

\section{Cell-viability test}

The viability of HCT116 and NCM460 cells treated with CM-SF NPs were evaluated with MTS assay (CellTiter 96; Promega, Fitchburg, WI, USA). ${ }^{13,22}$ Briefly, samples (CM-SF NPs, CM-dimethyl sulfoxide [DMSO], SF NPs, 5-Fu, and control at different concentrations or at $\mathrm{IC}_{50}$ ) were sterilized for 30 minutes via ultraviolet light. HCT116 and NCM460 cells were cultivated in the 96 -well plates $\left(5 \times 10^{3}\right.$ cells/well) for 24 hours. On the next day, the cell medium was replaced by the sterilized samples in DMEM. After 2-6 days of incubation, the culture medium was removed and cells were washed three times with PBS, then 4 hours of reaction was initiated with $200 \mu \mathrm{L}$ MTS solution added. A microplate reader (model 680; Bio-Rad Laboratories Inc., Hercules, CA, USA) was used to measure absorbance values at 490 $\mathrm{nm}$. Cell viability was calculated as:

$$
\text { Cell viability }(\%)=\frac{\text { Absorbance of test cells }}{\text { Absorbance of control }} \times 100
$$

where the $\mathrm{OD}_{490}$ values of cells treated with different groups corresponded to the absorbance of test cells and the $\mathrm{OD}_{490}$ values of untreated cells reflected the absorbance of control cells.

\section{Cell-cycle and apoptosis measurement}

HCT116 cells $\left(10^{6}\right)$ were treated with CM-SF NPs, CMDMSO, 5-Fu, and control, collected after removing supernatant, and then resuspended with PBS. Treated cells were fixed and kept at $4^{\circ} \mathrm{C}$ for 12 hours in ethanol $\left(70 \%,-20^{\circ} \mathrm{C}\right)$. Afterward, cells were collected and resuspended in $500 \mu \mathrm{L}$ PBS, followed by staining with $50 \mu \mathrm{L}$ 7-ADD (7-amino- actinomycin D) and incubating at room temperature in the dark for 30 minutes. Finally, flow cytometry (FACSCanto II; BD Biosciences, San Jose, CA, USA) was used to test the stained samples.

For apoptosis measurement, cells were treated with CM-SF NPs, CM-DMSO, 5-Fu, and control for 24 hours at $\mathrm{IC}_{50}$. All treated cells $\left(10^{6}\right)$ were collected and washed twice in PBS. Ice-cold binding buffer $(500 \mu \mathrm{L})$ was used to resuspend treated cell aggregates. The cell suspension was stained with $5 \mu \mathrm{L}$ annexin V-phycoerythrin and $5 \mu \mathrm{L}$ 7-ADD, then incubated in the dark for 5 minutes and tested by flow cytometry.

\section{Statistical analysis}

Each experiment was performed in triplicate, and all data are presented as means $\pm \mathrm{SD}$. Statistical analysis was performed using one-way analysis of variance with the level of statistical significance set at $P<0.05$.

\section{Results}

\section{Physicochemical properties}

Surface morphologies of samples are shown in Figure 2A and $\mathrm{B}$. As can be seen, the irregular original CM powders showed uneven large particles $(>1 \mu \mathrm{m})$, while the regular spherical CM-SF NPs were much smaller in size $(<100 \mathrm{~nm})$. Chemical structures of samples were compared (Figure 2C). Characteristic peaks of $1,260,1,144,956$, and $820 \mathrm{~cm}^{-1}$ in original CM corresponded to $1,260,1,155,960$, and $828 \mathrm{~cm}^{-1}$ in CM-SF NPs, respectively. Meanwhile, major characteristic peaks of 1,648 $(\mathrm{C}=\mathrm{O}$ stretching vibration $), 1,531(\mathrm{~N}-\mathrm{H}$ stretching vibration), and $1,245 \mathrm{~cm}^{-1}(\mathrm{~N}-\mathrm{H}$ bending and $\mathrm{C}-\mathrm{N}$ stretching vibrations) in SF corresponded to 1,648, 1,531, $1,260 \mathrm{~cm}^{-1}$ in the CM-SF NPs, respectively, from which it can be concluded that $\mathrm{CM}$ was successfully incorporated into the SF nanoplatform via SEDS. Importantly, the solubility of incorporated CM was greatly improved over the original CM (Figure 2D-F).

\section{Cellular uptake activity}

For studying the intracellular transport potential of CM-SF NPs, qualitative intracellular uptake assays were performed, and both 0.5- and 6-hour treated cells were chosen for observation. As shown in Figure 3A, there was no clear green fluorescence found in the control group or the CM-DMSO group at the 0.5 -hour time point. Only slight green fluorescence was observed after 6 hours of treatment in the CM-DMSO group. By contrast, clear green fluorescence presented in the CM-SF NPs group at the 0.5 -hour time point and its intensity increased greatly after 6 hours of treatment, which indicates 

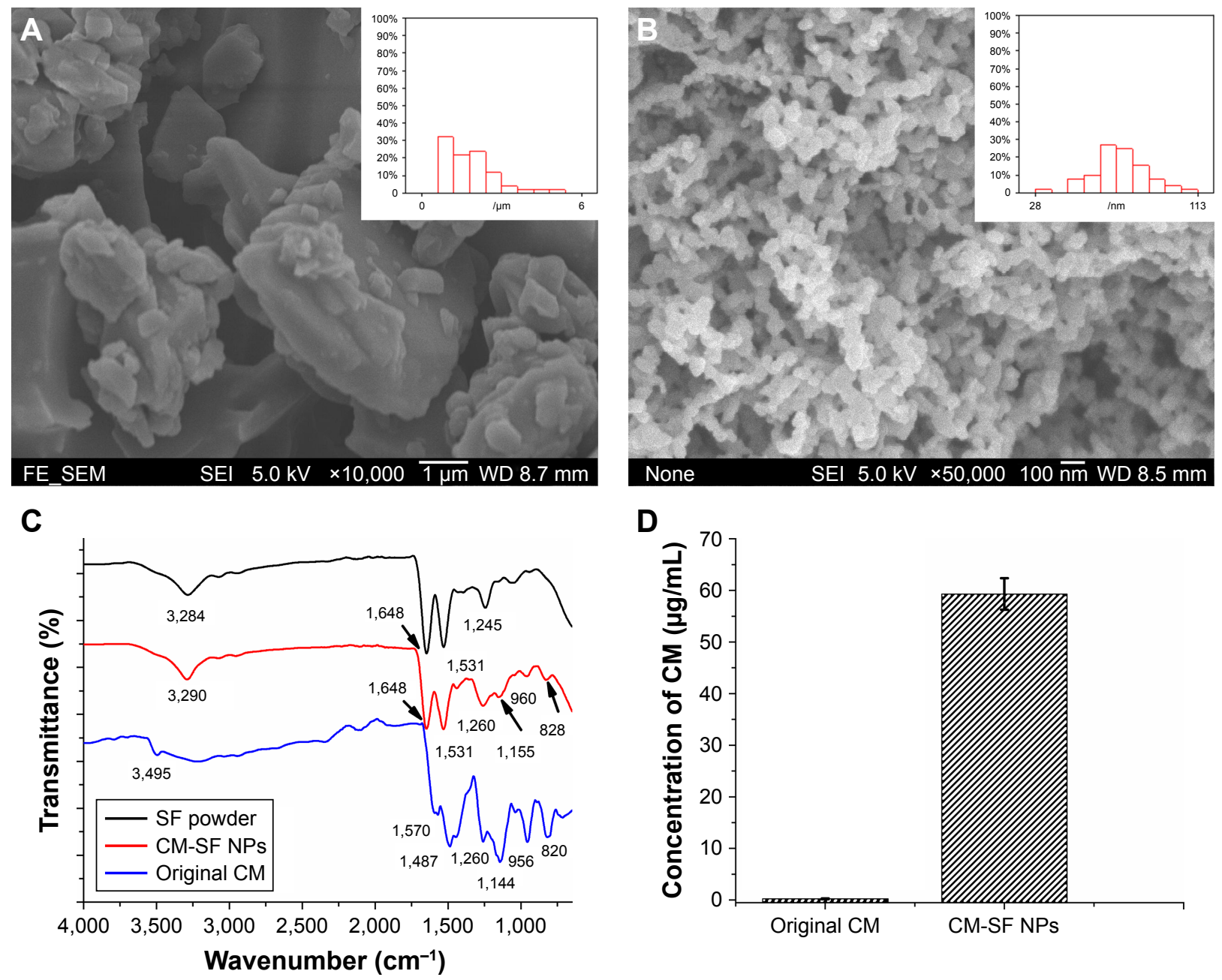

D
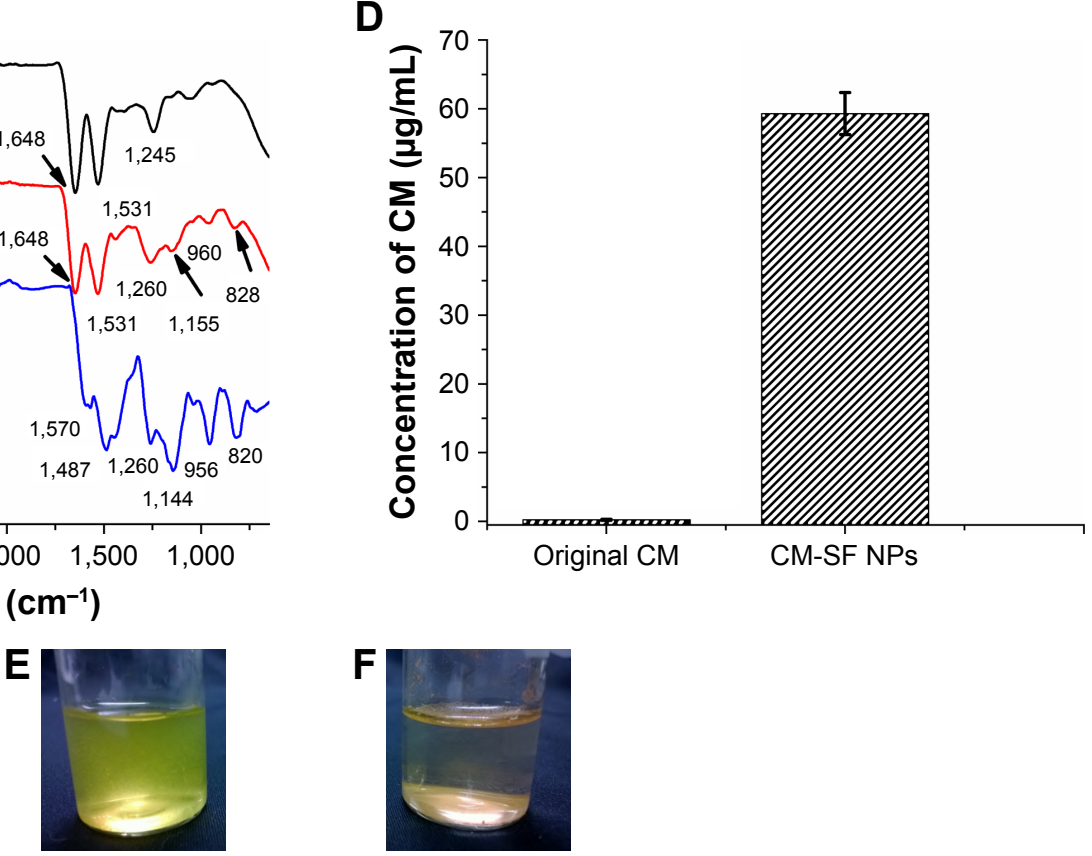

Figure 2 Physicochemical characterization of CM-SF NPs.

Notes: (A) SEM images of original CM powders (inset, particle size and distribution of original CM powders); (B) SEM images of CM-SF NPs prepared by SEDS CO ${ }_{2}$ (inset, particle size and distribution of CM-SF NPs); (C) FTIR spectra of SF, CM-SF NPs, and original CM powders; (D) solubility comparison of CM-SF NPs and original CM powders; (E, F) optical images of CM-SF NP solution and original CM-powder solution after I hour dissolution.

Abbreviations: CM, curcumin; SF, silk fibroin; NPs, nanoparticles; SEM, scanning electron microscopy; SEDS, solution-enhanced dispersion by supercritical CO ${ }_{2}$ FTIR, Fourier-transform infrared spectroscopy.

that CM-SF NPs had time-dependent intracellular transportability and showed nuclei-targeting potential as well. ${ }^{13,22}$

\section{Anticancer activity}

The cell viability of HCT116 was measured in response to CM-SF NPs, CM-DMSO, SF NPs, 5-Fu, and control treatments for 48 hours (Figure 4A). The inhibitory effect of 5-Fu improved with increased concentration $(0.5-20 \mu \mathrm{g} / \mathrm{mL})$. However, neither the CM-SF NP nor CM-DMSO group showed any inhibitory effect when concentrations were $<2 \mu \mathrm{g} / \mathrm{mL}$, but when concentration reached $5 \mu \mathrm{g} / \mathrm{mL}$, their anticancer effects sharply increased, maybe owing to the intrinsic property of CM. Meanwhile, CM-SF NPs had a stronger inhibitory effect $(\sim 68 \%)$ than CM-DMSO $(\sim 42 \%)$ group at $5 \mu \mathrm{g} / \mathrm{mL}$, and the $\mathrm{IC}_{50}$ value of CM-SF NPs group was lower compared to the CM-DMSO group (Table S1). CM-SF NPs presented superior anticancer potential ( $\sim 94 \%)$ compared to $5-\mathrm{Fu}(\sim 83 \%)$ when the concentration was higher than $10 \mu \mathrm{g} / \mathrm{mL}$. In contrast, cells treated with SF NPs retained about $90 \%$ viability at all tested 

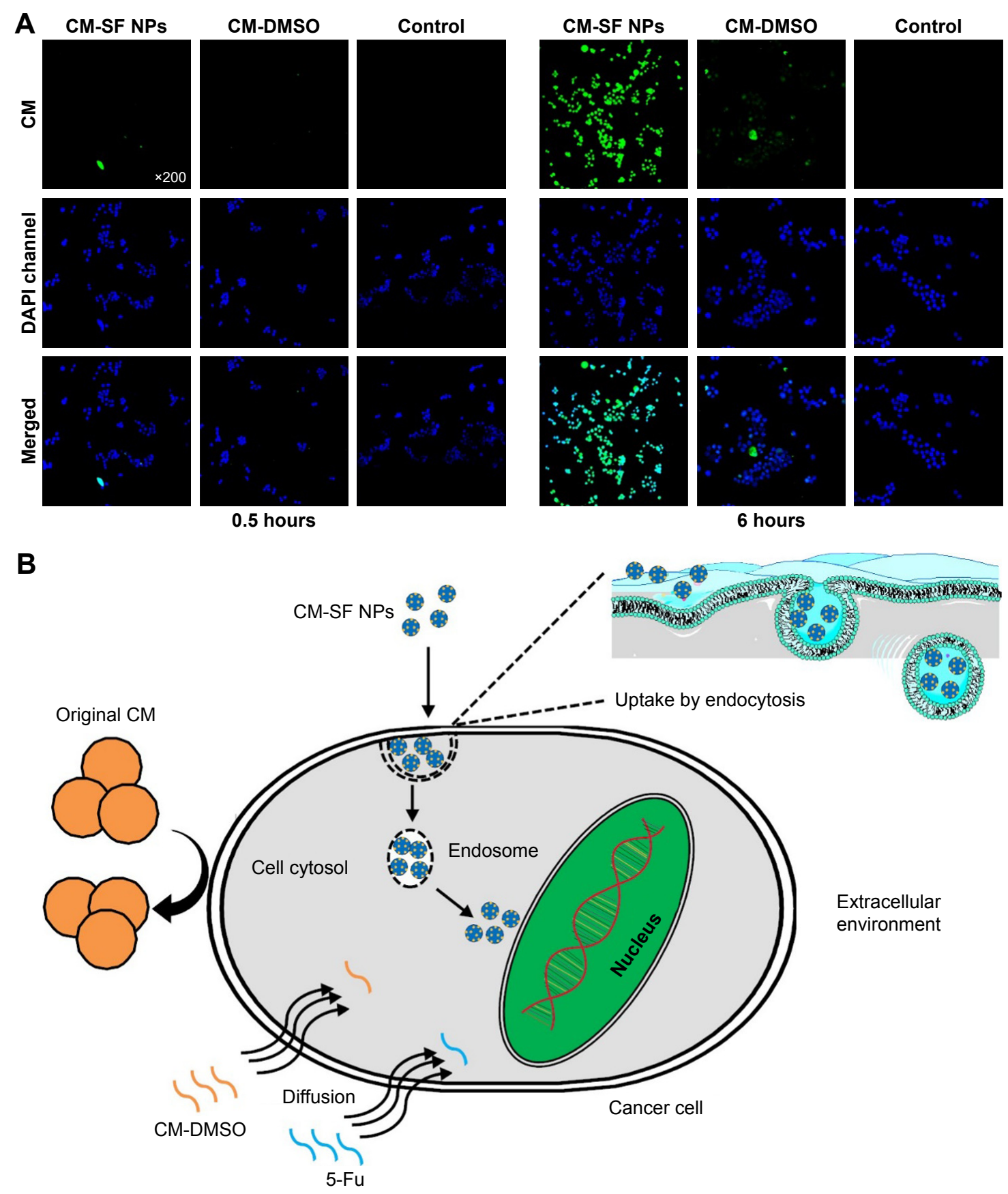

Figure 3 Fluorescence images and endocytosis pathway.

Notes: (A) Images show intracellular uptake efficiency of colon cancer cells (HCTI I6) after 0.5 and 6 hours' treatment with CM-SF NPs, CM-DMSO (original CM powders dissolved in DMSO solution), and control groups; (B) endocytosis pathway mechanism that enhanced cellular uptake efficiency of CM-SF NPs.

Abbreviations: CM, curcumin; SF, silk fibroin; NPs, nanoparticles; DMSO, dimethyl sulfoxide; DAPI, 4',6-diamidino-2-phenylindole.

concentrations. In summary, CM-SF NPs prepared by SEDS exhibited superior anticancer potential at concentrations higher than $5 \mu \mathrm{g} / \mathrm{mL}$.

\section{Cytotoxic activity}

It is necessary to evaluate the cytotoxicity of CM-SF NPs on normal human colon mucosal epithelial cells for potential biomedical applications. MTS results on NCM460 cells for concentrations of $0.1-200 \mu \mathrm{g} / \mathrm{mL}$ are shown in Figure 4B. As can be seen, 5-Fu exhibited toxic effects from $1 \mu \mathrm{g} / \mathrm{mL}$, while both CM-SF NPs and CM-DMSO exhibited toxic effects at $10 \mu \mathrm{g} / \mathrm{mL}$, and a significant difference in cell viability was observed ( $\sim 58 \%$ in CM-SF NPs and $\sim 14 \%$ in CM-DMSO), suggesting that the toxicity of CM incorporated 
A
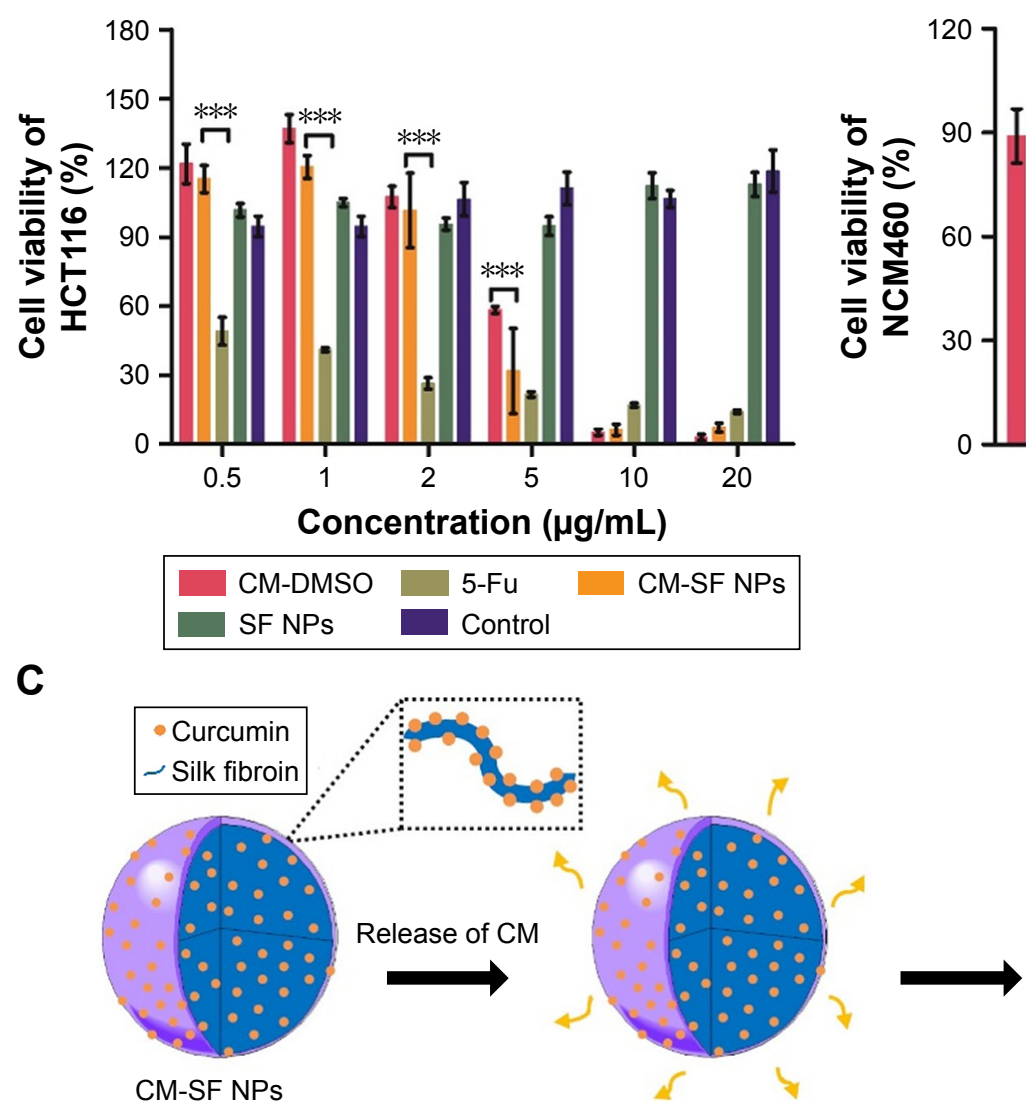

B
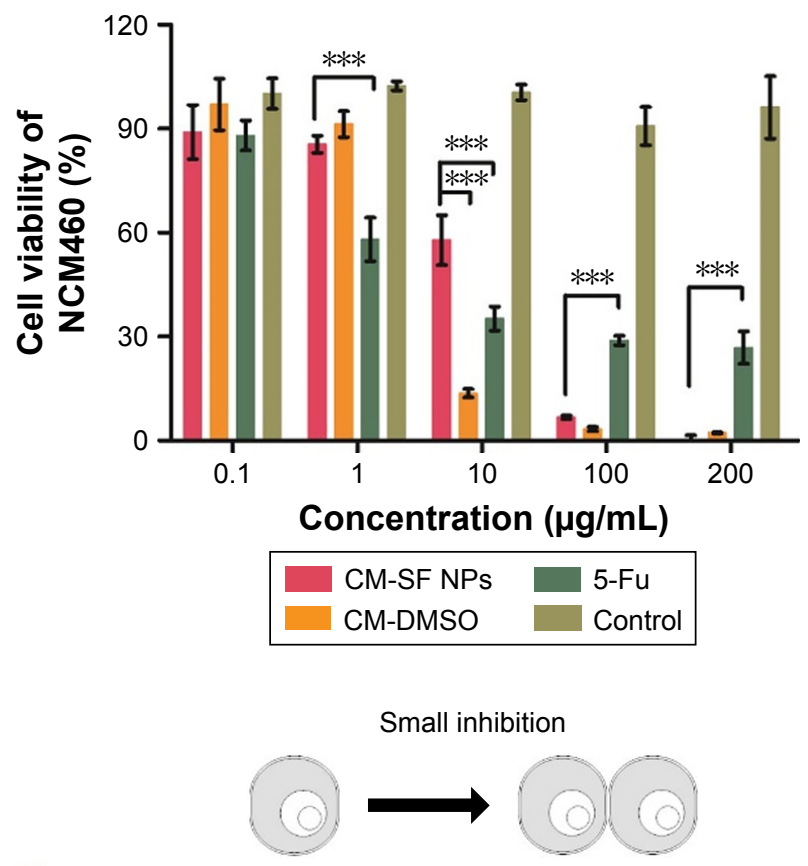

Normal cell

Smart-treatment concentration $(\sim 10 \mu \mathrm{g} / \mathrm{mL})$

Strong inhibition

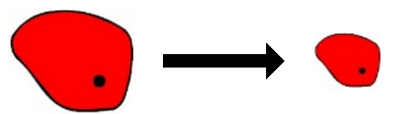

Cancer cell

Figure 4 Smart-treatment potential of CM-SF NPs on colon cancer cells.

Notes: (A) In vitro anticancer effect (dose-dependent) of CM-SF NPs on colon cancer HCTII 6 cells; (B) cytotoxicity of CM-SF NPs on normal NCM460 cells; (C) smarttreatment concentration range of CM-SF NPs, in which the growth of cancer cells was strongly inhibited, while the growth of normal cells was minimally inhibited. Results are shown as means $\pm S D, n=6$. $* * * P<0.001$.

Abbreviations: CM, curcumin; SF, silk fibroin; NPs, nanoparticles; DMSO, dimethyl sulfoxide; 5-Fu, fluorouracil.

into SF nanoplatforms was reduced at an effective concentration in the treatment of colon cancer.

\section{Mechanism of anticancer effect}

\section{Cell-cycle arrest}

To elucidate the mechanism of the inhibitory effect on colon cancer cells of CM-SF NPs, cells were treated for 24 hours at $\mathrm{IC}_{50}$. Results of cell-cycle distributions are shown in Figure $5 \mathrm{~A}$ and C. 5-Fu arrested high populations ( $(51 \%)$ of cells in the $\mathrm{G}_{2} / \mathrm{M}$ phase, while both CM-DMSO and $\mathrm{CM}$ NPs presented high percentages of cells in the $\mathrm{G}_{0} / \mathrm{G}_{1}(\sim 57 \%$ and $\sim 51 \%$, respectively) and $\mathrm{G}_{2} / \mathrm{M}$ phases ( $18 \%$ and $\sim 19 \%$, respectively) compared to the control group ( $\sim 7 \%$ and $\sim 12 \%$, respectively). Therefore, the inhibitory mechanism on colon cancer cells of CM-SF NPs was presumably due to enhanced efficacy in cell-cycle arrest at the $\mathrm{G}_{0} / \mathrm{G}_{1}$ and $\mathrm{G}_{2} / \mathrm{M}$ phases.
Induction of cell apoptosis

It is believed that cell apoptosis may represent a mechanism to counteract neoplastic development, which is important for cancer therapy. Reports on cancer therapy in recent years have revealed that $\mathrm{CM}$ can cause apoptosis signals in various cancers. ${ }^{23-25}$ As shown in Figure 5B and D, cells treated with CM-SF NPs showed a higher percentage of apoptosis $(\sim 23 \%)$ than those treated with 5-Fu ( 16\%). This indicates that CM-SF NPs had better apoptotic cell-induction potential than 5-Fu. In summary, the mechanism of inhibitory effect on colon cancer cells of CM-SF NPs was mainly due to cell-cycle arrest in the $\mathrm{G}_{0} / \mathrm{G}_{1}$ and $\mathrm{G}_{2} / \mathrm{M}$ phases in association with inducing apoptosis.

\section{Discussion}

$\mathrm{CM}$ is recognized as a potential chemotherapeutic drug. However, its clinical application is severely hindered by 

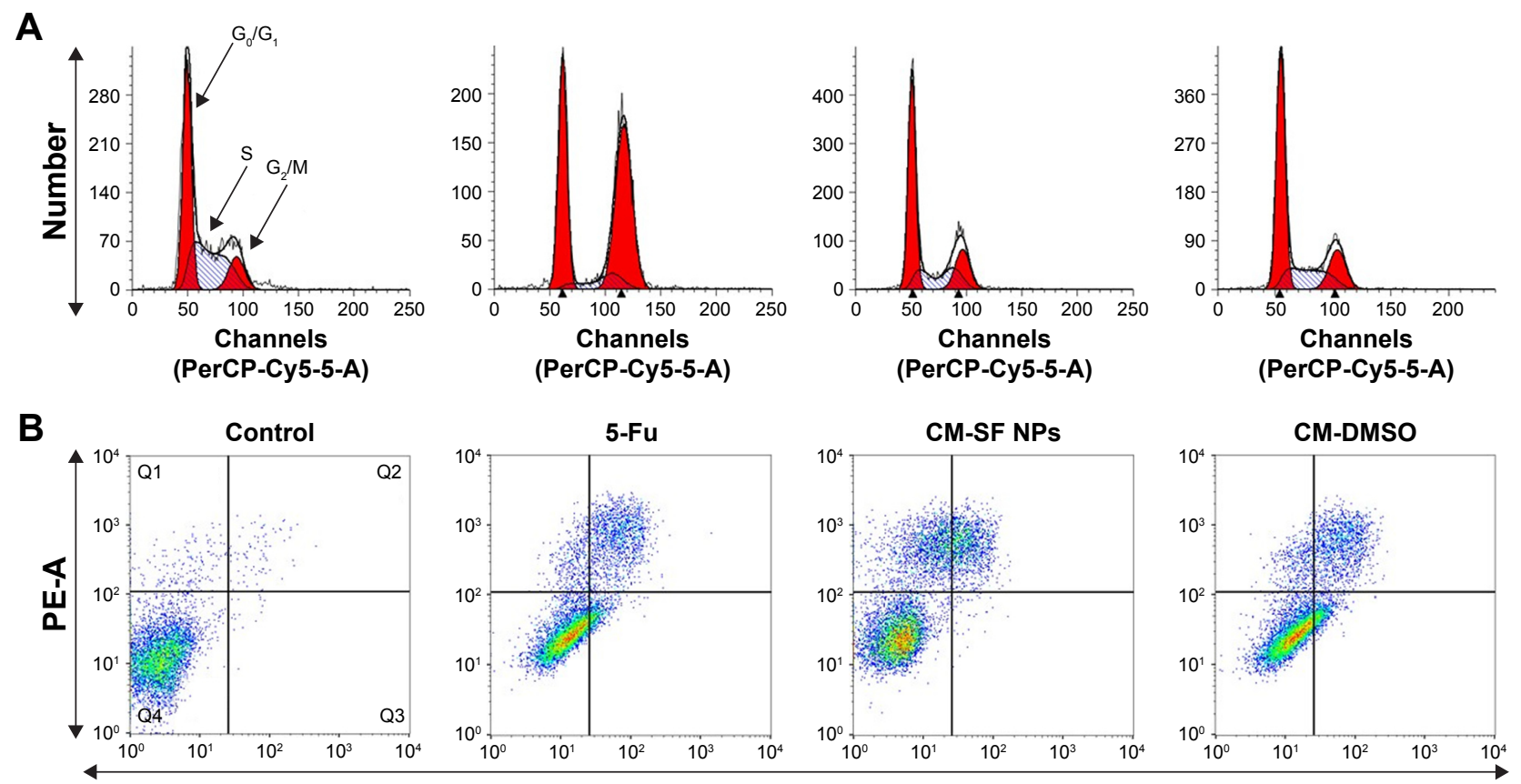

PerCP-Cy5-5-A
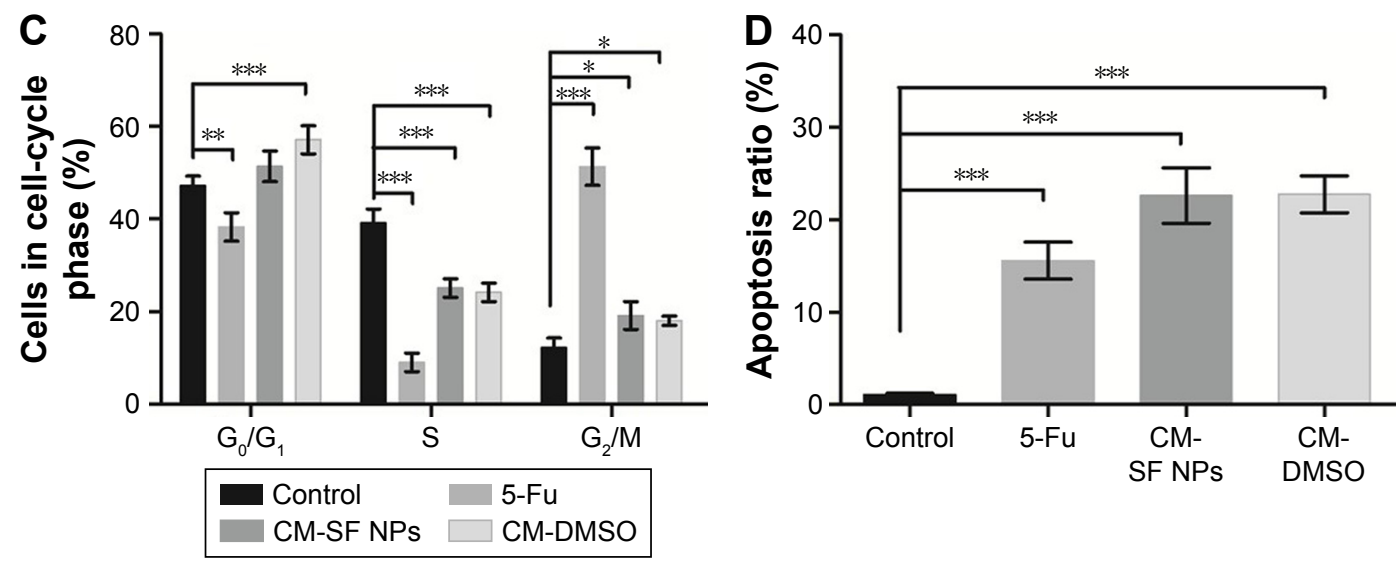

Figure 5 Anticancer mechanism of CM-SF NPs against colon cancer cells.

Notes: (A) Qualitative cell-cycle and (B) apoptotic progression of HCTII6 cells in response to control, 5-Fu, CM-SF NPs, and CM-DMSO treatment for 24 hours. Quadrants Q1, Q2, Q3, and Q4 reflect necrosis, late apoptosis, and early apoptosis and live, respectively. (C) Quantitative analysis of cell-cycle and (D) FACS distributions (\%) of apoptotic HCTII 6 cells in response to different groups at 24 hours. Total apoptosis includes late apoptosis plus early apoptosis. All data were obtained from at least three independent experiments. $* P<0.05 ; * * P<0.01 ; * * * P<0.001$.

Abbreviations: CM, curcumin; SF, silk fibroin; NPs, nanoparticles; DMSO, dimethyl sulfoxide; FACS, fluorescence-activated cell sorting; 5-Fu, fluorouracil.

poor solubility, low bioavailability, lack of bioavailability to tumors, and poor intracellular uptake efficiency. In our previous report, we prepared CM-SF NPs by using SEDS for the first time, and their solubility was remarkably increased over the original CM. ${ }^{9}$ Here, we further studied the controlled drug-release properties of SF nanoplatforms for smart colon cancer therapy.

In this study, small $(<100 \mathrm{~nm})$ spherical CM-SF NPs were prepared by SEDS. The chemical structure of CM-SF NPs was confirmed by FTIR results; it is believed that the incorporated CM most likely interacted with the hydrophobic residues in the silk nanoplatforms..$^{22,26}$ Moreover, the $3,495 \mathrm{~cm}^{-1}(\mathrm{O}-\mathrm{H})$ peak in $\mathrm{CM}$ shifted to $3,290 \mathrm{~cm}^{-1}$, suggesting that $\mathrm{CM}$ linked to the $\mathrm{SF}$ nanoplatforms, maybe via hydrogen bonds. ${ }^{19}$ Importantly, the solubility of CM-SF NPs was greatly improved, which was mainly due to size reduction, supersaturation of $\mathrm{CM}$, water solubility of the SF nanoplatform, better dispersibility and wettability of $\mathrm{CM}$, and hydrogen bonds between $\mathrm{CM}$ and the SF nanoplatform. ${ }^{27,28}$

The intracellular uptake result of CM-SF NPs is in agreement with previous studies. ${ }^{26,29}$ In those studies, the introduction of SF improved the retention of loaded drugs in cancer cells, thus leading to a higher anticancer efficacy. In our opinion, there are two reasons for the better cellular uptake 
efficiency of CM-SF NPs: 1) the endocytosis pathway, which is believed to be energy-costly for NP uptake (Figure 3B), and 2) the SF nanoplatforms are able to facilitate intracellular uptake.

In our opinion, the combination of improved intracellular uptake efficiency and enhanced permeability and retention effect of CM-SF NPs contributes to the higher toxic effect on colon cancer cells. Moreover, it is important to ascertain whether their anticancer effects would be retained or diminished during a therapeutic period. The cell viability of the treated cells (HCT116) for 6 days (at the $\mathrm{IC}_{50}$ of samples) was calculated (Figure S3). The results showed that CM-SF NPs and CM-DMSO had similar cell viability on days 2 and 4. However, a significant difference in cell viability was obtained on day 6, CM-SF NPs displaying an improved anticancer effect $(>98 \%)$ compared to CM-DMSO $(\sim 66 \%)$ and 5 -Fu $(\sim 63 \%)$, due to the sustained-release property of CM-SF NPs. Moreover, SF NPs did not display significant toxic effects. Therefore, CM-SF NPs possess both dose- and time-dependent anticancer effects, mainly due to sustained release. Our findings are similar to those of previous studies on colon cancer therapy by mucoadhesive CM-containing chitosan NPs. ${ }^{27,30}$

Meanwhile, CM-SP NPs showed reduced toxicity on normal human colon mucosal epithelial cells at an effective concentration $(\sim 10 \mu \mathrm{g} / \mathrm{mL})$ in the treatment of colon cancer cells. This phenomenon could be explained by the slow and sustained drug-release potentials of SF nanoplatforms, thereby reducing their cytotoxicity. Our observation is in agreement with an earlier report on colon cancer therapy with CM-poly(lactic-co-glycolic acid) (PLGA) conjugates. ${ }^{28,31}$ In conclusion, $\mathrm{sc}-\mathrm{CO}_{2}$-developed SF nanoplatforms showed a smart colon cancer therapy potential (Figure 4C).

The cell-cycle results indicated that the inhibition effect on colon cancer cells by CM-SF NPs was partly owing to cell-cycle arrest in the $\mathrm{G}_{0} / \mathrm{G}_{1}$ and $\mathrm{G}_{2} / \mathrm{M}$ phases. Our findings are in line with previous studies on doxorubicin and CM encapsulated in liposomes for tumor therapy ${ }^{29,32}$ and CMPLGA NPs for breast cancer treatment. ${ }^{30,33}$ Our cell apoptosis results were similar to the previous $\mathrm{CM}$ nanoformulation study. ${ }^{34}$ In the present study, we hypothesized that the better apoptosis induction ability of CM-SF NPs was owing to their higher cellular uptake efficiency, resulting in greater accumulation of released CM within cells in conjunction with continuous delivery that arrested a greater percentage of apoptotic cells. This result was also consistent with the MTS assays (Figure 4A). It has been reported that inhibition of the key antiapoptotic proteins Mcll and BclxL and induction of PARP cleavage are possible mechanisms of apoptosis induction by $\mathrm{CM} .{ }^{35}$

\section{Conclusion}

In conclusion, CM-SF NPs with controllable particle size $(<100 \mathrm{~nm})$ were prepared by SEDS in this study, with results showing that they showed time-dependent intracellular uptake ability due to SF nanoplatforms facilitating endocytosis, which led to improved inhibition effects on colon cancer cells. The mechanism can be explained by cell-cycle arrest in the $\mathrm{G}_{0} / \mathrm{G}_{1}$ and $\mathrm{G}_{2} / \mathrm{M}$ phases in association with inducing apoptotic cells. Interestingly, the anticancer effect of CM-SF NPs was improved while the side effect was reduced at concentrations of $\sim 10 \mu \mathrm{g} / \mathrm{mL}$, which may have been caused by the combination of the slow-release property of the SF nanoplatform and intrinsic properties of CM. In conclusion, NC-loaded SF nanoplatforms prepared by SEDS showed promising colon cancer therapy potential.

\section{Acknowledgments}

The authors acknowledge funding support from the University of Manchester (project codes AA14512 and AA01906), Guangdong Provincial Department of Science and Technology, China (project 2012B091000143), Guangdong Department of Finance, China (project 2014SC111), Science and Technology Program of Guangzhou, China (project 201607010157), and the Foundation for Distinguished Young Talents in Higher Education of Guangdong, China (project 2014KQNCX124).

\section{Disclosure}

The authors report no conflicts of interest in this work.

\section{References}

1. Li YY, Zhang T. Targeting cancer stem cells by curcumin and clinical applications. Cancer Lett. 2014;346(2):197-205.

2. Naksuriya O, Okonogi S, Schiffelers RM, Hennink WE. Curcumin nanoformulations: a review of pharmaceutical properties and preclinical studies and clinical data related to cancer treatment. Biomaterials. 2014;35(10):3365-3383.

3. Martin RC, Locatelli E, Li Y, et al. Gold nanorods and curcumin-loaded nanomicelles for efficient in vivo photothermal therapy of Barrett's esophagus. Nanomedicine (Lond). 2015;10(11):1723-1733.

4. Li SL, Fang CS, Zhang JQ, et al. Catanionic lipid nanosystems improve pharmacokinetics and anti-lung cancer activity of curcumin. Nanomedicine. 2016;12(6):1567-1579.

5. Mirzaei H, Shakeri A, Rashidi B, Jalili A, Banikazemi Z, Sahebkar A. Phytosomal curcumin: a review of pharmacokinetic, experimental and clinical studies. Biomed Pharmacother. 2017;85:102-112.

6. Margulis K, Magdassi S, Lee HS, Macosko CW. Formation of curcumin nanoparticles by flash nanoprecipitation from emulsions. $J$ Colloid Interface Sci. 2014;434:65-70. 
7. Santo IE, Campardelli R, Albuquerque EC, Vieira De Melo SA, Reverchon E, Della Porta G. Liposomes size engineering by combination of ethanol injection and supercritical processing. J Pharm Sci. 2015; 104(11):3842-3850.

8. Zhang J, Peng L, Han B. Amphiphile self-assemblies in supercritical $\mathrm{CO}_{2}$ and ionic liquids. Soft Matter. 2014;10(32):5861-5868.

9. Xie MB, Li Y, Zhao Z, et al. Solubility enhancement of curcumin via supercritical $\mathrm{CO}_{2}$ based silk fibroin carrier. J Supercrit Fluids. 2015; 103:1-9.

10. Xie MB, Li Y, Zhao Z, Chen AZ. Application of supercritical fluid technique in preparation of active protein microparticles. Poster presented at: Sixth Textile Bioengineering and Informatics Symposium; September 26-28, 2013; Xi'an, China.

11. Chen AZ, Chen LQ, Wang SB, Wang YQ, Zha JZ. Study of magnetic silk fibroin nanoparticles for massage-like transdermal drug delivery. Int J Nanomedicine. 2015;10:4639-4651.

12. Zhao Z, Xie MB, Li Y, et al. Formation of curcumin nanoparticles via solution-enhanced dispersion by supercritical $\mathrm{CO}_{2}$. Int $J$ Nanomedicine. 2015;10:3171-3181

13. Xie MB, Fan DJ, Zhao Z, et al. Nano-curcumin prepared via supercritical: improved anti-bacterial, anti-oxidant and anti-cancer efficacy. Int J Pharm. 2015;496(2):732-740.

14. Yoon IS, Park JH, Kang HJ, et al. Poly(D,L-lactic acid)-glycerol-based nanoparticles for curcumin delivery. Int J Pharm. 2015;488(1-2): 70-77.

15. Fang XB, Zhang JM, Xie X, et al. pH-sensitive micelles based on acid-labile Pluronic F68-curcumin conjugates for improved tumor intracellular drug delivery. Int J Pharm. 2016;502(1-2):28-37.

16. Kim S, Diab R, Joubert O, Canilho N, Pasc A. Core-shell microcapsules of solid lipid nanoparticles and mesoporous silica for enhanced oral delivery of curcumin. Colloids Surf B Biointerfaces. 2016;140: $161-168$.

17. Zhu BW, Wang H, Leow WR, et al. Silk fibroin for flexible electronic devices. Adv Mater. 2016;28(22):4250-4265.

18. Zhou ZT, Shi ZF, Cai XQ, et al. The use of functionalized silk fibroin films as a platform for optical diffraction-based sensing applications. Adv Mater. 2017;29(15):1605471.

19. Li G, Li Y, Chen GQ, et al. Silk-based biomaterials in biomedical textiles and fiber-based implants. Adv Healthc Mater. 2015;4(8):1134-1151.

20. Seib FP, Coburn J, Konrad I, et al. Focal therapy of neuroblastoma using silk films to deliver kinase and chemotherapeutic agents in vivo. Acta Biomater. 2015;20:32-38.

21. Li H, Tian J, Wu AQ, Wang JM, Ge CC, Sun ZL. Self-assembled silk fibroin nanoparticles loaded with binary drugs in the treatment of breast carcinoma. Int J Nanomedicine. 2016;11:4373-4380.

22. Xie MB, Fan DJ, Chen YF, et al. An implantable and controlled drugrelease silk fibroin nanofibrous matrix to advance the treatment of solid tumour cancers. Biomaterials. 2016;103:33-43.
23. Singh A, Kim W, Kim Y, et al. Multifunctional photonics nanoparticles for crossing the blood-brain barrier and effecting optically trackable brain theranostics. Adv Funct Mater. 2016;26(39):7057-7066.

24. Wang J, Wang Y, Liu Q, et al. Rational design of multifunctional dendritic mesoporous silica nanoparticles to load curcumin and enhance efficacy for breast cancer therapy. ACS Appl Mater Interfaces. 2016;8(40):26511-26523.

25. Yang X, Li ZJ, Wang N, et al. Curcumin-encapsulated polymeric micelles suppress the development of colon cancer in vitro and in vivo. Sci Rep. 2015;5:10322.

26. Rao W, Zhang WJ, Izmarie PF, et al. Thermally responsive nanoparticleencapsulated curcumin and its combination with mild hyperthermia for enhanced cancer cell destruction. Acta Biomater. 2014;10(2): 831-842.

27. Kanaujia P, Poovizhi P, Ng WK, Tan RB. Amorphous formulations for dissolution and bioavailability enhancement of poorly soluble APIs Powder Technol. 2015;285:2-15.

28. Wais U, Jackson AW, He T, Zhang H. Nanoformulation and encapsulation approaches for poorly water-soluble drug nanoparticles. Nanoscale. 2016;8(4):1746-1769.

29. Dong YX, Dong P, Huang D, et al. Fabrication and characterization of silk fibroin-coated liposomes for ocular drug delivery. Eur J Pharm Biopharm. 2015;91:82-90.

30. Chuah LH, Roberts CJ, Billa N, Abdullah S, Rosli R. Cellular uptake and anticancer effects of mucoadhesive curcumin-containing chitosan nanoparticles. Colloids Surf B Biointerfaces. 2014;116:228-236.

31. Waghela BN, Sharma A, Dhumale S, Pandey SM, Pathak C. Curcumin conjugated with PLGA potentiates sustainability, anti-proliferative activity and apoptosis in human colon carcinoma cells. PloS One. 2015; 10(2):e0117526.

32. Barui S, Saha S, Mondal G, Haseena S, Chaudhuri A. Simultaneous delivery of doxorubicin and curcumin encapsulated in liposomes of PEGylated RGDK-lipopeptide to tumor vasculature. Biomaterials. 2014; 35(5):1643-1656.

33. Verderio P, Bonetti P, Colombo M, Pandolfi L, Prosperi D. Intracellular drug release from curcumin-loaded PLGA nanoparticles induces G2/M block in breast cancer cells. Biomacromolecules. 2013; 14(3):672-682.

34. Chopra D, Ray L, Dwivedi A, et al. Photoprotective efficiency of PLGAcurcumin nanoparticles versus curcumin through the involvement of ERK/Akt pathway under ambient UV-R exposure in $\mathrm{HaCaT}$ cell line. Biomaterials. 2016;84:25-41.

35. Yallapu MM, Khan S, Maher DM, et al. Anti-cancer activity of curcumin loaded nanoparticles in prostate cancer. Biomaterials. 2014; 35(30):8635-8648. 


\section{Supplementary materials Formation of curcumin-silk fibroin nanoparticles}

The solution-enhanced dispersion by supercritical $\mathrm{CO}_{2}$ (SEDS) apparatus was composed of the following parts (Figure S1): a precipitation vessel for particle formation, a $\mathrm{CO}_{2}$ container and delivery part, and a solution transport and spray part. $\mathrm{CO}_{2}$ was cooled to $0^{\circ} \mathrm{C}$ and then pumped into a precipitation vessel, which was placed in a heated gas bath.

In the SEDS process, $\mathrm{CO}_{2}$ flow was maintained to keep the system pressure stable when temperature and pressure reached the desired values. Then, an enhanced mixing effect between the curcumin (CM)-silk fibroin (SF) blend solution and supercritical $\mathrm{CO}_{2}$ resulted when they met within a specially designed coaxial nozzle. Subsequently, the mixed flow was sprayed into the precipitation vessel quickly. Afterward, a fresh $\mathrm{CO}_{2}$ flow was maintained to completely clear the HFIP residue. Then, the precipitation vessel was depressurized slowly. Finally, dry CM-SF nanoparticles were obtained and vacuum-dried, then stored at $4{ }^{\circ} \mathrm{C}$ for further characterization.

\section{Surface morphology, particle size, and size distribution}

An aluminum sample holder with a thin self-adherent carbon film was used to load the samples, and then they were coated with a thin layer of gold. The surface morphology of

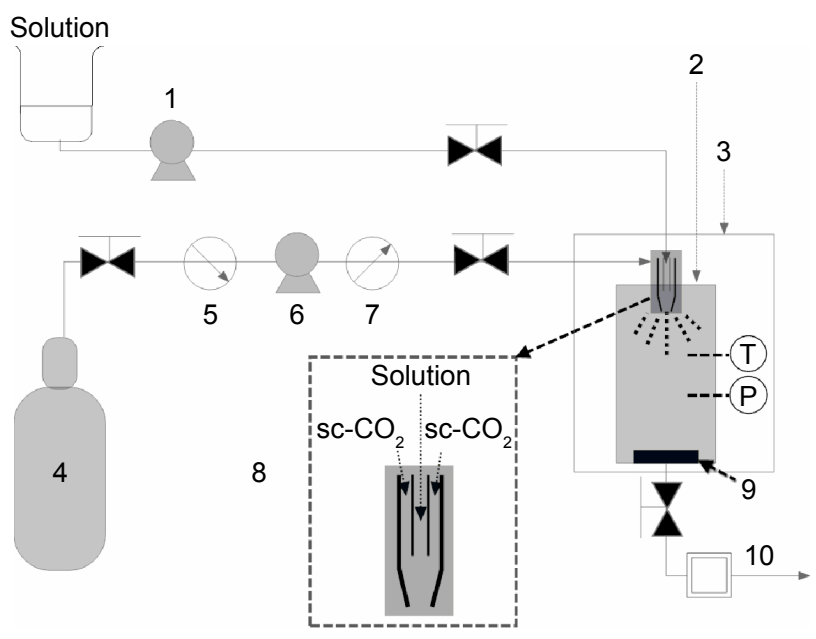

Figure SI Solution-enhanced dispersion by sc- $\mathrm{CO}_{2}$ equipment.

Notes: I, Solution pump; 2, precipitation vessel; 3, gas bath; 4, $\mathrm{CO}_{2}$ cylinder; 5 , cooler; $6, \mathrm{CO}_{2}$ pump; 7 , heat exchanger; 8 , specially designed coaxial nozzle; 9 , gas filter; 10, water bath.

Abbreviations: P, pressure gauge; sc, supercritical; T, thermometer.

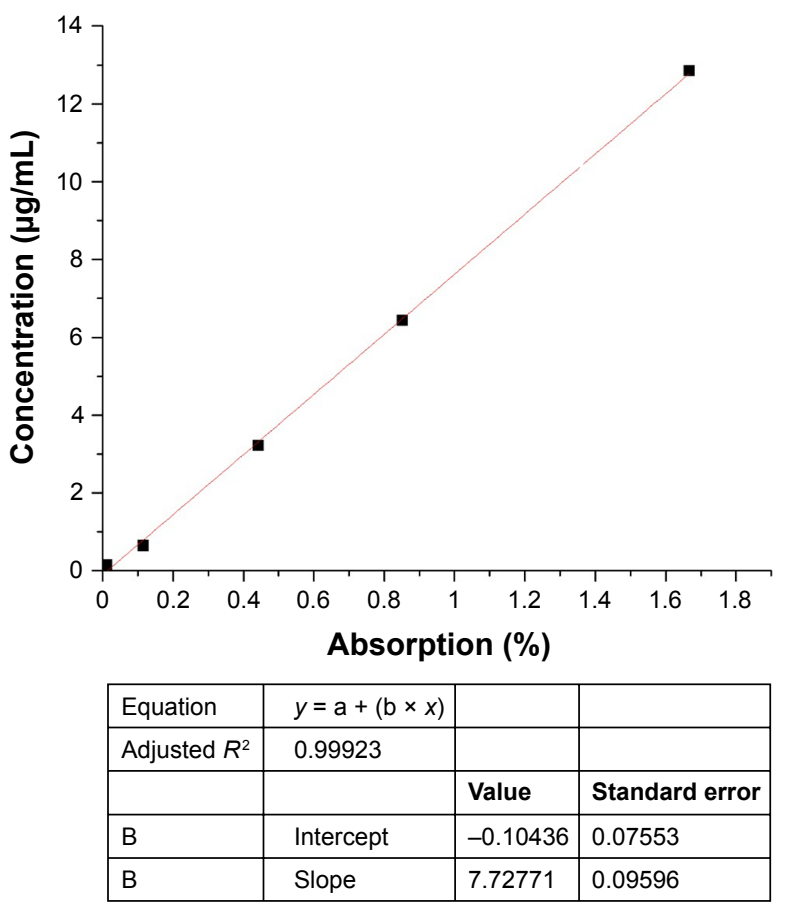

Figure S2 Standard curve of curcumin.

the samples was visualized by using field-emission scanning electron microscopy (SEM, JSM-6490; JEOL, Tokyo, Japan). The equivalent diameters of 500 particles in the obtained SEM photographs were measured, and particle size and size distribution were analyzed by Nano Measurer software.

\section{Physicochemical characterization}

$\mathrm{KBr}$ powders were used to mix the obtained samples (ratio 1:100), and then the mixtures were transformed to a thin tablet. Fourier-transform infrared spectra of the obtained samples were collected and analyzed with a 1720 spectrophotometer (PerkinElmer, Waltham, MA, USA) in transmission mode with the wave number ranging $400-4,000 \mathrm{~cm}^{-1}$. For solubility test results, see Figure S2.

\section{Cell culture and proliferation}

Both NCM460 and HCT116 cells were incubated in RPMI 1640 medium (Thermo Fisher Scientific, Waltham, MA,

Table SI IC ${ }_{50}$ comparison of samples

\begin{tabular}{ll}
\hline & IC $_{50}(\mu \mathrm{g} / \mathrm{mL})$ \\
\hline CM-DMSO & 5.339 \\
CM-SF NPs & 4.383 \\
5 -Fu & 0.432 \\
\hline
\end{tabular}

Abbreviations: CM, curcumin; DMSO, dimethyl sulfoxide; SF, silk fibroin; NPs, nanoparticles; 5-Fu, 5-flurouracil. 


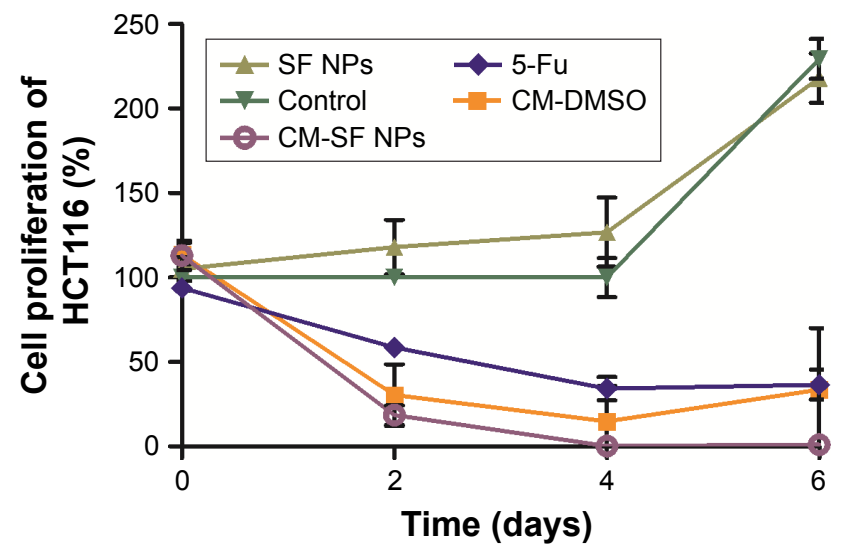

Figure S3 Cell proliferation of HCTII6 after treatment with CM-SF NPs, CMDMSO, 5-Fu, SF NPs, and control.

Note: Results are shown as means $\pm S D, n=6$.

Abbreviations: CM, curcumin; SF, silk fibroin; NPs, nanoparticles; DMSO, dimethyl sulfoxide; 5-Fu, 5-flurouracil.
USA) combined with $1 \%$ penicillin-streptomycin $(100 \mathrm{U} / \mathrm{mL}$ penicillin and $100 \mu \mathrm{g} / \mathrm{mL}$ streptomycin; Thermo Fisher Scientific) and $10 \%$ fetal bovine serum (Thermo Fisher Scientific). Cells were cultured in an incubator with a humidified atmosphere of $37^{\circ} \mathrm{C} \pm 0.2^{\circ} \mathrm{C}$ and $5 \% \mathrm{CO}_{2}$.
International Journal of Nanomedicine

\section{Publish your work in this journal}

The International Journal of Nanomedicine is an international, peerreviewed journal focusing on the application of nanotechnology in diagnostics, therapeutics, and drug delivery systems throughou the biomedical field. This journal is indexed on PubMed Central, MedLine, CAS, SciSearch ${ }^{\circledR}$, Current Contents ${ }^{\circledR} /$ Clinical Medicine,

\section{Dovepress}

Journal Citation Reports/Science Edition, EMBase, Scopus and the Elsevier Bibliographic databases. The manuscript management system is completely online and includes a very quick and fair peer-review system, which is all easy to use. Visit http://www.dovepress.com/ testimonials.php to read real quotes from published authors.

\footnotetext{
Submit your manuscript here: http://www.dovepress.com/international-journal-of-nanomedicine-journal
} 